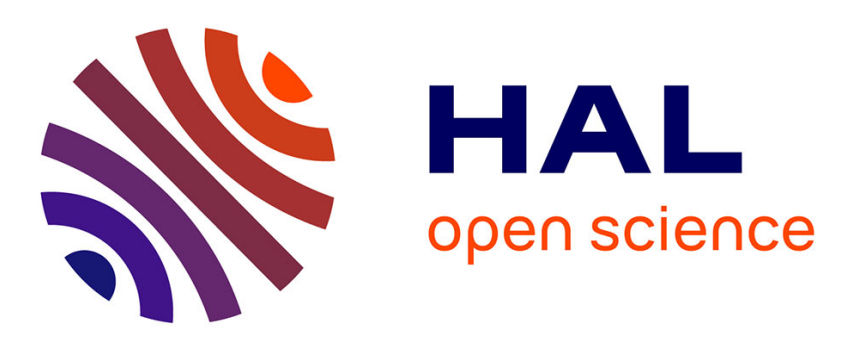

\title{
Crowd Navigation in VR: exploring haptic rendering of collisions
}

Florian Berton, Fabien Grzeskowiak, Alexandre Bonneau, Alberto Jovane, Marco Aggravi, Ludovic Hoyet, Anne-Hélène Olivier, Claudio Pacchierotti, Julien Pettré

\section{To cite this version:}

Florian Berton, Fabien Grzeskowiak, Alexandre Bonneau, Alberto Jovane, Marco Aggravi, et al.. Crowd Navigation in VR: exploring haptic rendering of collisions. IEEE Transactions on Visualization and Computer Graphics, 2022, 28 (7), pp.2589 - 2601. 10.1109/TVCG.2020.3041341 . hal-03038344

\section{HAL Id: hal-03038344 https://hal.inria.fr/hal-03038344}

Submitted on 3 Dec 2020

HAL is a multi-disciplinary open access archive for the deposit and dissemination of scientific research documents, whether they are published or not. The documents may come from teaching and research institutions in France or abroad, or from public or private research centers.
L'archive ouverte pluridisciplinaire HAL, est destinée au dépôt et à la diffusion de documents scientifiques de niveau recherche, publiés ou non, émanant des établissements d'enseignement et de recherche français ou étrangers, des laboratoires publics ou privés. 


\title{
Crowd Navigation in VR: Exploring Haptic Rendering of Collisions
}

\author{
Florian Berton, Fabien Grzeskowiak, Alexandre Bonneau, Alberto Jovane, Marco Aggravi, Ludovic Hoyet, \\ Anne-Hélène Olivier, Claudio Pacchierotti, Julien Pettré
}
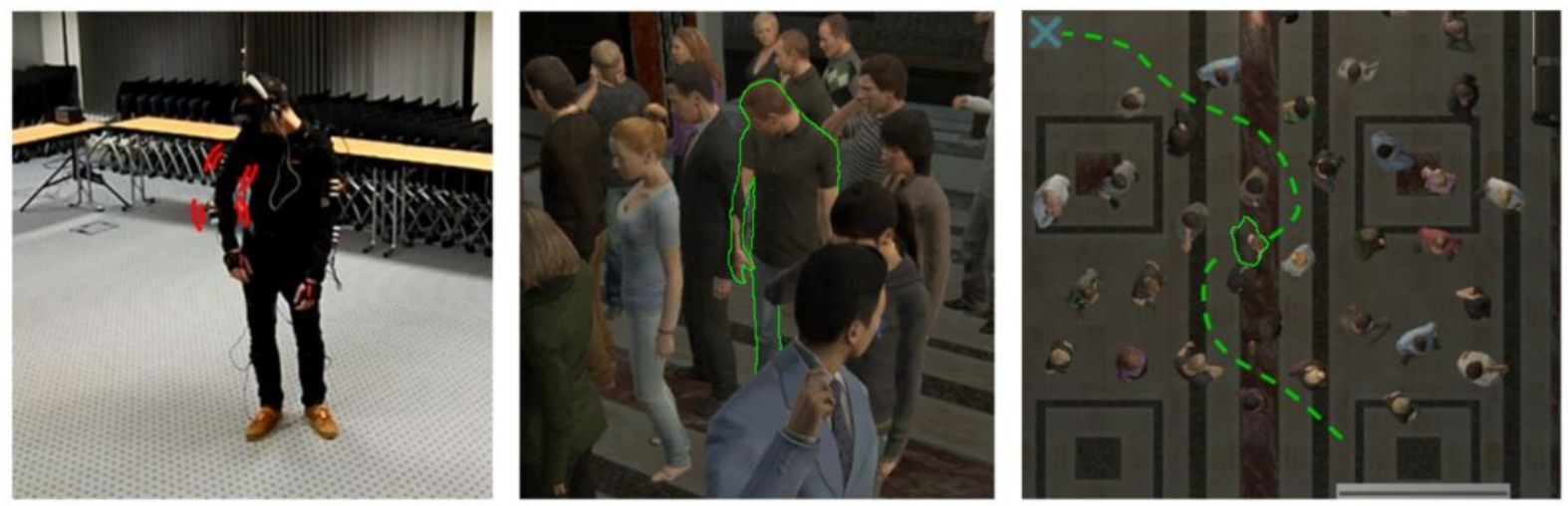

Fig. 1. Our objective is to understand whether and to what extent providing haptic rendering of collisions during navigation through a virtual crowd (right) makes users behave more realistically. Whenever a collision occurs (center), armbands worn on the arms locally vibrate to render this contact (left). We carried out an experiment with 23 participants, testing both subjective and objective metrics regarding the users' path planning, body motion, kinetic energy, presence, and embodiment.

\begin{abstract}
Virtual reality (VR) is a valuable experimental tool for studying human movement, including the analysis of interactions during locomotion tasks for developing crowd simulation algorithms. However, these studies are generally limited to distant interactions in crowds, due to the difficulty of rendering realistic sensations of collisions in VR. In this work, we explore the use of wearable haptics to render contacts during virtual crowd navigation. We focus on the behavioural changes occurring with or without haptic rendering during a navigation task in a dense crowd, as well as on potential after-effects introduced by the use haptic rendering. Our objective is to provide recommendations for designing VR setup to study crowd navigation behaviour. To this end, we designed an experiment $(\mathrm{N}=23)$ where participants navigated in a crowded virtual train station without, then with, and then again without haptic feedback of their collisions with virtual characters. Results show that providing haptic feedback improved the overall realism of the interaction, as participants more actively avoided collisions. We also noticed a significant after-effect in the users' behaviour when haptic rendering was once again disabled in the third part of the experiment. Nonetheless, haptic feedback did not have any significant impact on the users' sense of presence and embodiment.
\end{abstract}

Index Terms-Crowd, Human interaction, Haptic rendering, Virtual Reality

\section{INTRODUCTION}

Virtual Reality (VR) offers interesting opportunities for the study of human behaviours, including the possibility of accurately controlling experimental conditions, reproducing them across different subjects and trials, and easily accessing environmental information. For example, we use VR in crowd modelling and simulation for analysing the dynamics of local interactions between individuals in crowded environments [9].

To achieve good crowd models and simulations, we need to very well capture human behaviour. However, this is not an easy task, since human behaviour relies on many different variables and covers a wide range of interactions. Moreover, when running crowd behaviour experiments in

Inria, Univ Rennes, M2S, CNRS, IRISA, France e-mail: firstname.lastname@inria.fr
VR, there is a high chance that participants will behave differently from how they behave in real life. This mismatch is due to different reasons, most of them linked to the limited sense of realism that VR provides. In this respect, significant efforts have been put in the evaluation of the realism of behaviours in VR [16], [33], in the understanding of the visual information required [31] or in the development of highly-realistic VR environments and characters [1]. However, despite these efforts, most VR experiences still lack of any haptic sensation, which is of course of paramount importance when studying crowd behaviour and interactions. For example, if we are unable to render the sensation of bumping into virtual characters when navigating in a crowded environment, participants might stop avoiding collisions, leading to data that does not capture well how humans truly behave.For this reason, 
studies of collective behaviour in VR are often limited to cases considering distant interactions only [38], [39], so as not to require any haptic feedback.

This paper explores the role of contact interactions (collisions) during navigation in a crowded environment. To do so, we employ a set of wearable haptic interfaces able to provide compelling vibrotactile sensations of contact to the user's arms. Our objective is to investigate whether and to what extent the rendering of contacts influences the user's behaviour in this context, as well as limits the occurrence of certain well-known artifacts, such as when the user's virtual avatar interpenetrates other virtual characters. We conducted an experiment $(\mathrm{N}=23)$ where participants were equipped with four wearable haptic interfaces (two on each arm), and asked to navigate in a densely-crowded virtual train station. We evaluate objective metrics related to the user's behaviour with respect to the crowd, as well as subjective metrics related to the user's sense of presence and embodiment. First, we carried out the experiment without haptic rendering of contacts, then with haptic rendering, and finally once again without haptic rendering. This experimental design enables us to register the difference in user's behaviour when activating the haptic feedback as well as the persistence of any relevant after-effect. These results are expected to help all researchers planning to use VR for studying human behaviour when navigating a crowded environment.

The rest of the paper is structured as follow. Section 2 summarizes the state of the art on the topic, comparing what has been presented in the literature to what we are proposing in this paper. Then, Section 3 describes the experimental setup, methods, and task. Section 4 presents the metrics we considered, based on the study of local body movements, trajectories, energy, contacts, embodiment, and presence. Section 5 discusses the results and analyses the differences between the considered conditions using inferential statistical analysis methods. Section 6 discusses our findings as well as their implications for crowd experiments in VR. Finally, Section 7 draws the final remarks and discusses future work on the topic.

\section{Related Work}

\subsection{Pedestrian Interaction Behaviours}

Understanding how pedestrians interact is a fundamental requirement for the design of realistic crowd simulators. To that purpose, several studies have been conducted in real conditions to investigate collective behaviours. For example, Seyfried et al. [44] have shown that speed depends on density in a crowd (i.e., fundamental diagram) and this relation is affected by cultural aspects [10]. Bonneaud et al. [6] showed that, without any instruction, collective behaviour can emerge within a group of walkers and this behaviour can be described according to several patterns, such as the anisotropy of interpersonal distance or speed synchronization. Some other studies focused on the local aspects of interactions. Using pairwise situations, authors previously showed that pedestrians adapt their motion only if there is a future risk of collision [35]. Authors considered the effect of situational factors such as crossing angle [24], [25], crossing order [34] or orientation [8] as well as personal factors such as gender and personality [25] or body size [8] on motion adaptations. While these previous studies have considered the kinematics of the adaptions, other works were interested in the gaze activity, showing that it can predict future crossing order [17] and that gaze behaviour is task-dependent [22].

Nevertheless, interactions between humans are multifactorial and challenging to fully control and standardize for studying purposes. To tackle this issue, recent works have proposed to use virtual reality to design new experiments on person-person interactions. A lot of effort has been put to validate this tool, both for the study of trajectory [33], [45] and gaze [4] behaviours. Studies agree that virtual reality is very promising for conducting such experiments, since the nature of the interactions is preserved and participants are expected to behave in the same way as in real conditions. However, some quantitative differences have been observed, e.g., an increase of the crossing distance and larger head movements, which can be due to the distorted perception of distances and limitations of the field of view introduced by VR head-mounted displays. Nevertheless, VR opens large perspectives in the design of new experiments for understanding pedestrian behaviours. For examples, having only one participant at the same time, researchers were able to evaluate the effect of specific factors such as emotions of the crowd on participant behaviours and proxemics [7], [23], [49] or the effect of density on gaze behaviour [3], user experience [19] as well as navigation strategies when facing a group of moving people (i.e., going around or going through the group) [9].

While the interest of virtual reality is widely established for the study of interactions between pedestrians, studies in VR have mainly designed experimental paradigms which involved vision, proprioception as well as the vestibular system to perceive user actions and their surroundings in the virtual environment. It is worth to note that these studies did not provide haptic feedback to the users, which appears as an important input when dealing with person-person interactions and detection of collisions with other walkers, especially when navigating in dense crowds. Our paper can be seen as a continuation of previous work that explored realism of locomotion trajectories [16], [33] under various conditions of VR setup designs, while we now consider the effect of haptic rendering.

\subsection{Virtual Realty and Collision Rendering}

Immersive VR must actively engage one's senses to make the human user feel truly part of the virtual world. This feeling of immersion is achieved through multiple types of features and information that flow from the virtual environment to the human user. Rich sensory feedback is of course a fundamental pillar of this feeling of immersion in VR, which includes the rendering of virtual contacts. Pacchierotti et al. [36] presented a review paper on wearable haptic devices used to render contact sensations at the fingertip and hand. Notable examples of this technology used for VR applications are [12], [14], [41], [43]. Collisions are of course not limited to the hand, especially when dealing with crowded environments. In this respect, Lindeman et al. [28], [29] developed a wearable haptic vest and belt capable 
of providing distributed vibrotactile feedback sensations in VR. Vibrations were used to indicate a contact in the virtual environment or to provide information about areas of the environment yet to explore. More recently, Mestre et al. [32] performed an experiment where participants had to walk through a door with a variable width in VR with or without haptic rendering or $3 \mathrm{~d}$ body representation. Haptic rendering was a vibrotactile feedback at the shoulders notifying users when they were in close vicinity of the door structure (from $10 \mathrm{~cm}$ until contact). Participants showed the same basic behaviour (shoulder rotation when passing a narrow door) with and without haptic rendering or body representation. Furthermore, the optimal behaviour to avoid collision with the door's borders required the presence of body representation and vibrotactile feedback. Louison et al. [30] tested wearable vibrotactile feedback in an industrial VR training application. Participants wore a haptic sleeve on their right arm, which provided vibrotactile feedback sensations via ten vibrating motors. Users had to activate or deactivate a series of targets in VR, following a given order. Contacts between the environment and the user's arm were rendered by vibrotactile feedback, where trials with vibrotactile feedback showed a higher spatial awareness and less contacts with the environment. Bimbo et al. [5] employed eight vibrotactile motors in two armbands to provide the user with feedback information about the collisions between a robotic arm and the cluttered environment it operated in. A similar work was presented recently in [2]. Regarding interaction with virtual characters, Krogmeier et al. [26] designed an experiment where participants had to bump into a virtual character, with or without haptic rendering of contacts. This haptic rendering was performed using the "Tactsuit"1 , equipped with 70 haptic points of contact. In this preliminary study, they showed that this kind of haptic feedback improves presence and embodiment. In another context, Krum et al. [27] were interested in the impact of different locomotion techniques and priming haptic rendering on proxemics and subjective social measures during interaction with a virtual character. The priming haptic rendering corresponded to a simulated touch by the virtual human. Their results showed that priming haptic rendering did not influence participant's proxemics but influenced the subjective social measures. For instance, it improved the sympathy and the relation toward the virtual character. Furthermore, Faure et al. [20] asked participants to perform a collision avoidance task with a virtual character, while walking on a treadmill. They used a cable mechanism to render physical contacts, which increased the minimal distance between the participant and the virtual character, as well as made participants tend to initiate the avoidance strategy sooner.

All these studies focus on the interaction with one virtual character. However, to the best of our knowledge, no study designed an experiment where participants had to navigate in a dense virtual crowd while wearing haptic rendering devices. The use of tactile feedback therefore raises questions about their effect on participants' behaviour, which sets the objective of our study, as described in the next section.

1. https://www.bhaptics.com/

\section{Experimental Overview}

The purpose of this study is to investigate the effect of haptic rendering of collisions on participants' behaviour during navigation through a static crowd in VR. To explore this question, we immersed participants in a virtual train station and asked them to perform a navigation task which involved moving through a crowd of virtual characters. In some conditions, collisions with the virtual characters were rendered to participants using 4 wearable vibrotactile haptic devices (actuated armbands). Our general hypothesis is that haptic rendering changes the participants' behaviour by giving them feedback about the virtual collisions. Moreover, we also expect that even after removing haptic rendering, an after-effect still persists on the participants' behaviour.

\subsection{Materials \& Methods}

\subsubsection{Apparatus}

For the purpose of immersing participants in the virtual environment and investigating the potential effects of haptic rendering while navigating in groups of characters, we used the following devices, which are summarized in Figure 2:

- Motion Capture: to record participants' body motions, as well as to render their animated avatar in the scene, we used an IMU-based (Inertial Measurement Unit) motion capture system $\left(\mathrm{Xsens}^{2}\right)$. The Xsens system provides real-time, easy-of-use, reliable and accurate human motion tracking. IMU sensors were equipped on the participants using motion capture suit and straps, while body tracking was handled by the Xsens MVN Animate software and streamed to Unity in real time.

- HMD: to immerse participants in the virtual environment, we chose to use a $\operatorname{Pimax}^{3}$ virtual reality headset, in particular because of the wide field of view provided in these situations of close proximity with other characters (specifications: $90 \mathrm{~Hz}, 200^{\circ}$ fov, $2560 \times 1440$ resolution). The HMD was used with 4 SteamVR 2.0 base stations, providing a tracking area of approximately $10 \times 10 \mathrm{~m}$. This setup enabled participants to physically walk in the real space, while their walking movements were displayed on their avatar in the size-matched virtual environment.

- Haptic Rendering: to render haptic collisions between participants and the virtual characters, we equipped participants with four armbands (one on each arm and forearm) [42]. Each armband is composed of four vibrotactile motors with vibration frequency range between 80 and $280 \mathrm{~Hz}$ and controlled independently. Motors are positioned evenly onto an elastic fabric strap (see Figure 3). An electronics board controls the hardware. It comprises a $3.3 \mathrm{~V}$ Arduino Mini Pro, a $3.7 \mathrm{~V}$ Li-on battery, and a Bluetooth 2.1 antenna for wireless communication with the external control station.

- Computer: to let participants move freely in the environment, they were equipped with a MSI VR One backpack computer, which was running the

2. https://www.xsens.com/

3. https://www.pimax.com/ 

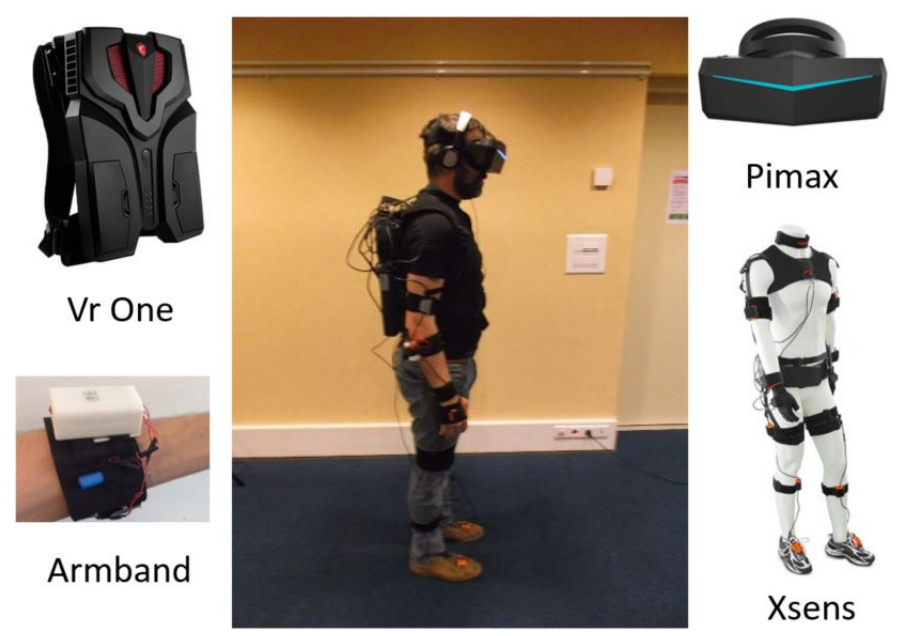

Pimax

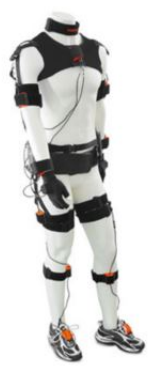

Xsens

Fig. 2. Devices worn by participants during the experiment.

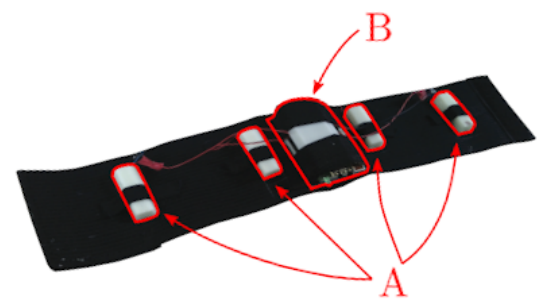

Fig. 3. Wearable vibrotactile armband, composed of four vibrating motors (A). The electronics is enclosed in a 3D-printed case (B) [42].

experiment. All the devices were connected directly to this computer (specifications: NVidia GTX 1070, Intel Core i7-7820HK processor, 32 GB RAM).

\subsubsection{Haptic rendering}

Haptic rendering requires collisions to be detected in the VR environment. Since haptic devices were worn on participant's arms, we detected collisions between their avatar (animated using the Xsens motion capture system) and the virtual crowd. To this end, we segmented each avatar's arm into three parts (arm, forearm, and hand), and attached to each segment a Unity capsule collider that reported on collisions with other objects in the scene (see Figure 4). When a collision was detected, that is if one of the six segments of the avatar entered in collision with the geometry of any virtual crowd character, one of the four haptic devices was activated. More specifically, colliders on the left (resp. right) virtual forearm and hand activated the armband located on participants' left (resp. right) forearm, while colliders on the left (resp. right) virtual upper arm activated the armband located on participants' left (resp. right) upper arm.

In terms of vibrations, each vibro-motor of an armband was driven using a single parameter called vibrotactile rate, which controlled both the amplitude and the frequency of vibration. During the experiment, all the motors of an activated armband were therefore controlled using the same vibrotactile rate, which varied according to a $10 \mathrm{~Hz}$-period sine wave profile. The variation of the vibrotactile rate resulted in a frequency of vibration in the range of [57126 ] Hz. Although these motors can vibrate up to $255 \mathrm{~Hz}$,

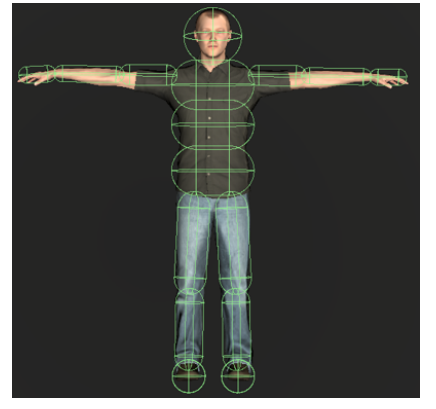

a)

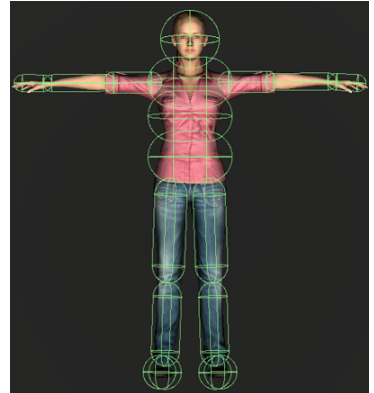

b)
Fig. 4. Male (a) and female (b) avatars used to represent the participants in the virtual environment. For both avatars the capsule around each segment represents the solid used to compute collisions.

we decided to limit their range after participants in a pilot study reported the full vibrating range to be too strong.

Communication with the armbands was performed at $4 \mathrm{~Hz}$, meaning that collisions with a duration lower than $250 \mathrm{~ms}$ were not rendered to participants, and that there was a maximum delay of $250 \mathrm{~ms}$ in activating (resp. stopping) the armbands after a collision was detected (resp. ended).

\subsection{Environment \& Task}

Participants were immersed in a digital reproduction of the metro station "Mayakovskaya" in Moscow, amongst a virtual static crowd (see Figure 5). A total of 8 different configurations of the scene were prepared in advance and used in the experiment. A configuration is defined by the exact position of each crowd character in the virtual station. In each configuration, the crowd formed a squared shape, and character positions followed a Poisson distribution resulting in a density of $1.47 \pm 0.06$ character $/ \mathrm{m}^{2}$. Such a distribution combined with such a level of density ensures that a gap of $0.60 \mathrm{~m}$ on average exists between each character. The crowd is composed of standing virtual characters animated with various idle animations (only small movement but standing in place). In each configuration, characters were animated according to two types of behaviour, either waiting (oriented to face the board displaying train schedules, moving slightly the upper body) or phone-calling (with a random orientation). We used several animation clips for each of the two behaviours, in order to prevent the exact same animation clip to be used for two different virtual characters.

At the beginning of each trial, participants were initially standing at one corner of the square crowd, embodied in a gender-matched avatar (see Figure 4). They were instructed to traverse the crowd so as to reach the board displaying train schedules, and to read aloud the track number of the next train displayed on the board before coming back to their initial position. They were physically walking in the real room, while their position and movements were used to animate their avatar. This task required participants to reach the opposite corner of the space in order to read information on the board, while forcing them to move through the virtual crowd. Also, the screen displayed the train information only when participants were at less than $2 \mathrm{~m}$ from it (i.e., when they reached the green area displayed 


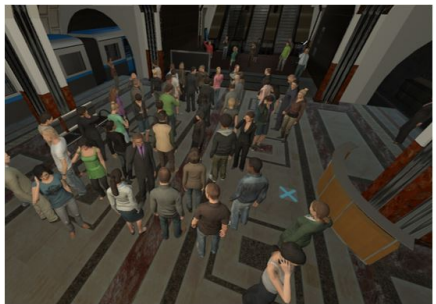

a)

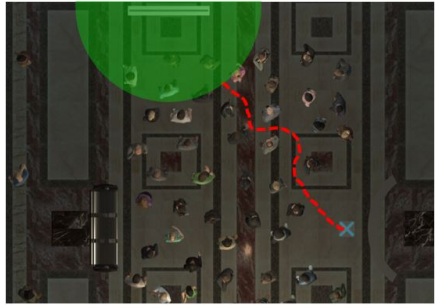

b)
Fig. 5. Snapshots of the environment under two different points of view. Participants started from the blue cross on the floor, and were instructed to reach the screen board. Figure (b) displays an example trajectory in a red doted line. The screen displayed the train information only when participants reached the green area.

in Figure 5.b). Furthermore, we provided the following instruction to participants prior to the experiment: "Walk through the virtual train station as if you were walking in a real train station".

\subsection{Protocol}

Upon arrival, participants were asked to fill in a consent form, during which they were presented the task to perform. They were then equipped with the equipment listed in Section 3.1.1. Calibration of the Xsens motion capture system was then performed to ensure motion capture quality, as well as to resize the avatar to participants dimensions. Once ready, participants performed a training trial in which they could explore the virtual environment and get familiar with the task.

The experiment then consisted of 3 blocks of 8 trials, where the blocks were presented for all participants in the following order: NoHaptic1, Haptic, and NoHaptic2. The Haptic block corresponded to performing the task with haptic rendering of contacts, while the NoHaptic blocks did not involve any haptic rendering of contacts. The experiment therefore consisted in performing first a block without haptic rendering, in order to measure a baseline of participants' reactions. The purpose of the second block was then to investigate whether introducing haptic rendering influenced their behaviour while navigating in a crowd, while the purpose of the last block (without haptic) was to measure potential after-effects. In each trial, participants performed the task described in Section 3.2 once. Each block was comprised of 8 trials, corresponding to the 8 crowd configurations presented in Section 3.2, performed in a random order. At the end of each block, participants were asked to answer the Embodiment and Presence questionnaires (Tables 2, 3, 4 \& 5) while remaining in the virtual environment. Finally, at the end of the experiment, participants filled in a demographic questionnaire.

\subsection{Participants}

Twenty-three unpaid participants, recruited via internal mailing lists amongst students and staff, volunteered for the experiment $(8 \mathrm{~F}, 15 \mathrm{M}$; age: avg $=26 \pm 6, \min =18, \max =43)$. They were all naive to the purpose of the experiment, had normal or corrected-to-normal vision, and gave written and informed consent. The study conformed to the declaration of Helsinki, and was approved by the Inria internal ethical committee (COERLE).

\subsection{Hypotheses}

$H 1$ : Haptic rendering will not change the path followed by participants through the crowd. Indeed, pedestrians mainly rely on vision to control their locomotion [37], [50], and we replicated each crowd configuration across the 3 blocks, resulting into identical visual information for participants to navigate. Therefore the followed path will be similar in the tree blocks of the experiment (NoHaptic1, Haptic and NoHaptic2).

$H 2$ : Haptic rendering of collisions will make participants aware of collisions and influence their body motion during the navigation through the crowd. Therefore, concerning the NoHaptic1 and Haptic blocks of the experiment, we expect that:

$H 2_{1}$ : Participants will navigate in the crowd more carefully in the Haptic block in order to avoid collisions. There will be more local avoidance movements (e.g., increased shoulder rotations) and a difference in participants' speed.

$H 2_{2}$ : With theses changes on participants' local body motions, there will be both less collisions, and smaller volumes of interpenetration when a collision occurs.

H3: We expect some after-effect due to haptic rendering, i.e., we expect that participants will remain more aware and careful about collisions even after we disabled haptic rendering. Therefore we expect $H 2_{1}$ and $H 2_{2}$ to remain true in the NoHaptic2 block.

H4: Haptic rendering will improve the sense of presence and the sense of embodiment of participants in VR, as they will become more aware of their virtual body dimensions in space with respect to neighbour virtual characters.

\section{Analysis}

This section presents the collected data as well as the variables used to evaluate our hypotheses.

\subsection{Collected Data}

During the experiment, we recorded at $45 \mathrm{~Hz}$ the trajectories of participants, as well as the position and orientation of their limbs in the virtual environment using the Xsens sensors and Unity. We also recorded the body poses over time of each character of the virtual crowd. Then, we were able to replay offline the entire trials in order to compute complex operations such as the volume of each collision.

\subsection{Trajectories}

To study $H 1$, we compared participants' trajectories through the virtual crowd. To this end, we decomposed the environment into cells based on a Delaunay triangulation [11], the vertices of which were the crowd characters. A trajectory is then represented as a sequence of traversed cells. An 


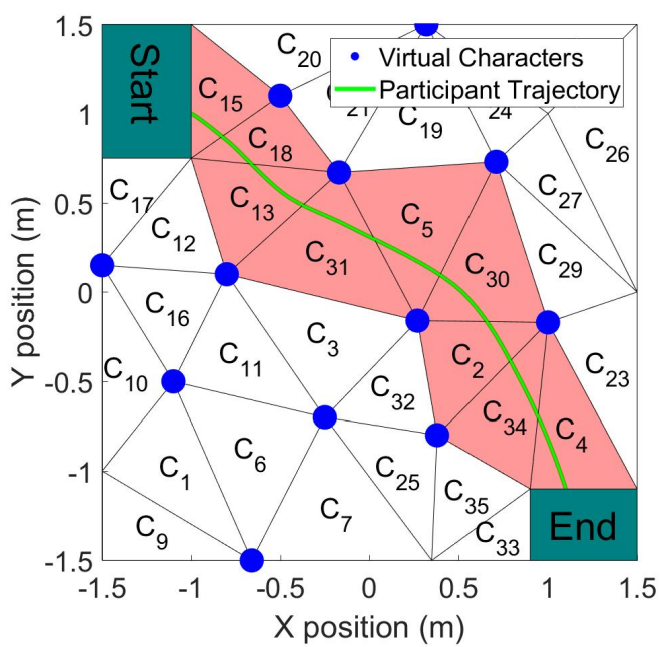

Fig. 6. Illustration of a participant's trajectory in a crowd, and the decomposition of the environment in cells using Delaunay triangulation [11].

example is displayed in Figure 6, where the displayed trajectory corresponds to the following sequence of cells: $C_{15} C_{18} C_{13} C_{31} C_{5} C_{30} C_{2} C_{34} C_{4}$.

Represented this way, comparison is possible only when the configuration of crowd characters is identical, which is one reason why we ensured to repeat the same configurations through the 3 studied blocks (cf. Section 3). In other words, we first grouped trajectories by crowd configuration, and then compared the set of trajectories performed in the same crowd configuration across different conditions.

Comparison was based on the Dice similarity coefficient (DSC) [47]. The DSC computes the similarity between two sets $A$ and $B$ according to:

$$
D S C(A, B)=\frac{2|A \cap B|}{|A|+|B|}
$$

Since our trajectories are sets of traversed cells, two trajectories traversing the same set of cells will be $100 \%$ similar. Similarity will decrease with the number of different cells traversed by the participant (occurring in one trajectory and not the other).

\subsection{Body Motions}

Navigating in cluttered environments, such as studied in this experiment, requires participants to weave with their body through the crowd. This section presents the data that will be used to analyse body movements when navigating through the virtual crowd to study $H 2_{1}$.

\subsubsection{Shoulder Rotation}

Turning the shoulders is a known strategy for squeezing through narrow openings [51], i.e., in our case to get between two close characters. To evaluate the effect of haptic rendering on the emergence of such behaviours we measured the shoulder orientation at certain critical points of the path. These critical points are the crossing points between the Delaunay cell boundaries (cf. Section 4.2) and the participant's trajectories.
More specifically, we computed the angle $\alpha_{S A}$ between the participants' shoulder-to-shoulder axis and the segment connecting the two considered virtual characters, as shown in Figure 7. This angle provides information about the orientation of the shoulders, and thus the trunk, at the narrowest parts of their path when participants passed between two characters. The larger this angle, the more careful - trying to lower their width at the maximum - participants were when traversing the opening between the two virtual characters.

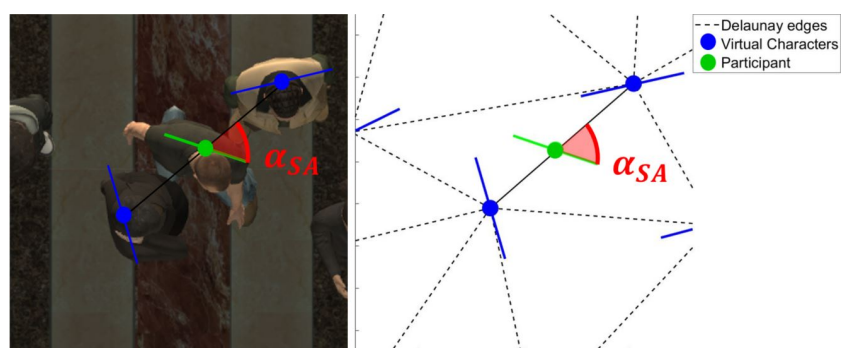

Fig. 7. Shoulder rotation. Angle $\alpha_{S A} \in[0,90]^{\circ}$ is defined between the participants' shoulder-to-shoulder axis and the segment connecting the two virtual characters. Left: top view of the scene. Right: diagram with the Delaunay triangles, the virtual characters, and the participant.

\subsubsection{Walking Speed}

Beyond the postural analysis introduced in the previous section, we are also interested in the walking speed to analyse whether participants performed the motion task differently according to conditions. To evaluate this parameter only during the navigation, we removed portions of trials where participants were mostly static (e.g., the time during which they were reading the board). To this end, we computed the minimum distance between the participant and the screen, which corresponds to the moment when participants stopped to read the information. We then removed all the frames when the participant's position was less than one step from this position (chosen as $0.74 \mathrm{~m}$ for men and $0.67 \mathrm{~m}$ for women [15]).

\subsection{Collisions}

A collision is the detected contact between any part of the participant's virtual body and any part of the mesh of one virtual character. We identify a collision by the pair participant-virtual character as well as the initial time. This means that we separately classify collisions with different characters, even if they are happening at the same time. This also means that we can detect several collisions with the same character but with different initial times. The detection starts at the first contact of any of the limbs of the character involved and the participant's geometry, and it lasts until there is no more contact detected between the two respective meshes. To analyse the collisions we selected two main values of interest: the number of collisions and the maximum volume of interpenetration between the participant and the virtual character during a collision:

- Number of collisions. We count any collision with an interpenetration volume greater than $10^{-6} \mathrm{~m}^{3}$ and lasting more than $10 \mathrm{~ms}$.

- Maximum volume of interpenetration. The maximum volume of interpenetration between a participant's 


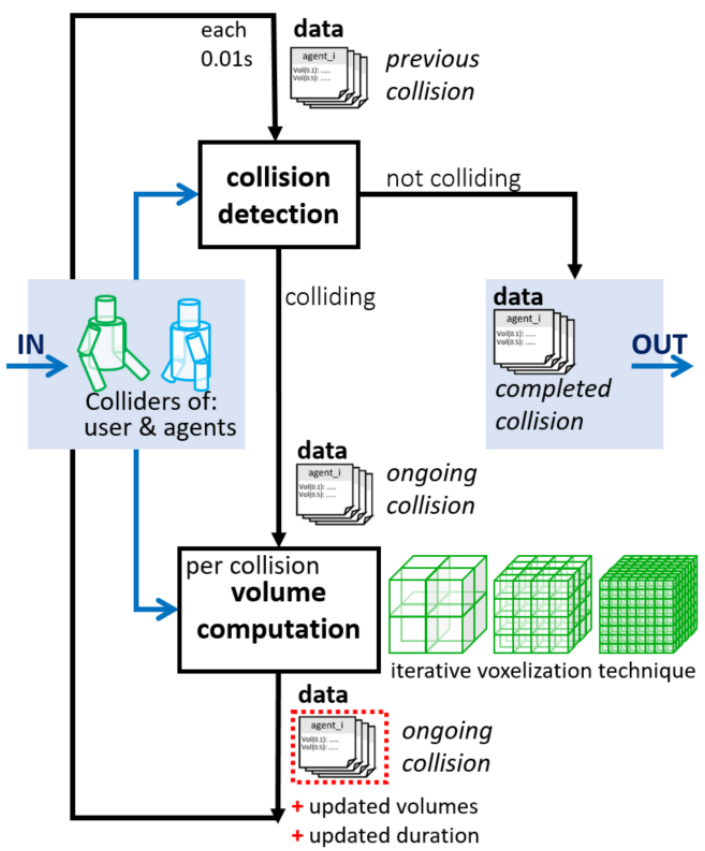

Fig. 8. Collision iteration loop scheme representing one step of the collision detection, in which we detect if there is a collision (either a new or an ongoing one) and compute its volume. We add this information to collision's data. When the collision is finished we send out the data.

avatar and a virtual character during a collision is computed at each time stamp through the voxelization of the intersection of their respective meshes, according to the following procedure. Each $10 \mathrm{~ms}$ the computation starts from the meshes of the two characters involved. Around those, we build an AABB (axis aligned bounding box), which is then iteratively subdivided in octant where, at each of this octantiteration, only the voxels in collision are kept. The octant-iteration stops when the target voxel size is reached. In our analysis, it was set to a cube of width $0.01 \mathrm{~m}$. This process is shown in Figure 9. At the end we collect all the volumes computed at each time interval of $10 \mathrm{~ms}$ and we extract the maximum one.

\subsection{Presence and Embodiment}

Another important aspect of our analysis is its perceptual relevance. In accordance with $H 4$, we looked for any difference in the users' feelings of presence and embodiment, comparing the registered subjective perception with and without haptic rendering. Participants answered both questionnaires at the end of each block (Embodiment then Presence), answering each question on a 7-point Likert scale.

\subsubsection{Presence}

Using an haptic device is generally expected to increase the user's immersion in the virtual world [26], as it adds a new sensorial feedback, even though it does not always lead to an increase of perceived realism [46]. For this reason, we measured Presence using the Slater-Usoh-Steed (SUS) questionnaire [48] (Table 5). Each user answered the set of 6 questions, summarized in Table 5, at the end of each block.

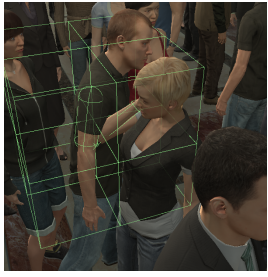

a)

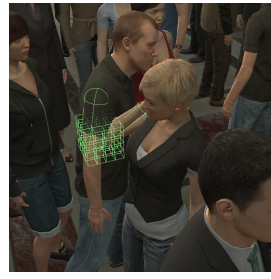

b)

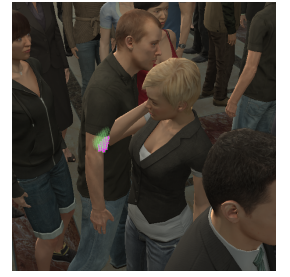

c)
Fig. 9. Volume computation using iteration of voxel spaces of decreasing dimensions. (a) Starting from the AABB around the selected geometries, the first voxel space with 8 voxels (green cubes) is created and intersected with the geometries. (b) In the next iteration only the intersecting voxels are kept, and further subdivided into 8 cubes each. (c) The process is iteratively applied until reaching the minimum subdivision size, where the final interpenetration volume is displayed in purple.

\subsubsection{Embodiment}

As for Presence, we focused on comparing the sense of embodiment between different blocks to study the influence of the haptic rendering on the perception of the virtual body. We measured embodiment based on the Roth and Latoschik questionnaire [40]. Participants answered Embodiment questionnaires simultaneously with those about Presence.

\subsection{Statistical Analyses}

Our objective is to understand whether and to what extent users change their behaviour in each experimental block. To do so, we analysed the differences across blocks for all the aforementioned variables.For all dependent variables, we set the level of significance to $\alpha=0.05$. First, a Shapiro-Wilk test was performed to evaluate whether the distribution of our data followed a normal distribution. If the distribution was not normal, a Friedman test was performed to evaluate the effect of the condition on these variables. Post-hoc comparisons were then performed using a Wilcoxon signed rank test with Bonferroni correction. On the other hand, if the distribution was normal, a one-way analysis of variance (ANOVA) with repeated measures was performed. Greenhouse-Geisser adjustments to the degrees of freedom were applied if the data violated the sphericity assumption. Bonferroni post-hoc tests were used to analyse any significant effects between groups.

\section{Results}

This section presents the results of our experiment, starting with the study of $H 1$ on the trajectories formed by participants through the virtual crowd. We then explore $H 2_{1}$ and $H 2_{2}$ with respect to the analysis of body movements. Finally, we report the results on collision metrics so as to evaluate $H 3$, to finish with the answers to the Presence and Embodiment questionnaires related to $H 4$.

\subsection{Trajectory Analysis}

Table 1 shows the results of the Dice similarity measure between all possible pairs of blocks. Similarity ranges from $84.7 \%$ (Nohaptic1 vs. Haptic blocks) to $88.5 \%$ (Haptic vs. NoHaptic2 blocks). The score is higher for Haptic 

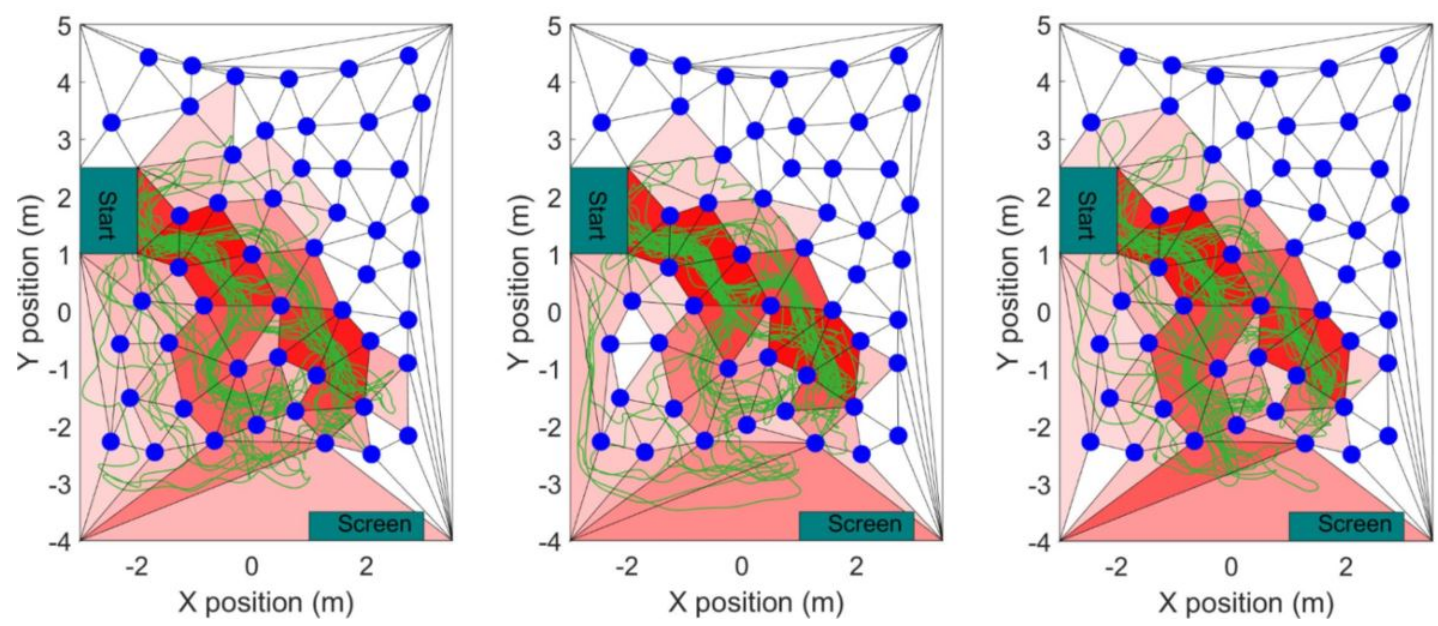

- Virtual Characters - Participant

$-24$

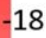

$-12$

$-6$

$-0$

Fig. 10. Participants' trajectories and Delaunay triangulation for trial $T_{6}$ for blocks NoHaptic1 (left), Haptic (middle) and NoHaptic2 (right). The color-bar represents the number of times participants walked on a triangle.

vs. Nohaptic2 blocks $(88.6 \pm 4.1 \%)$ and for Nohaptic1 vs. Nohaptic2 $(85.9 \pm 4.0 \%)$.

Because it is difficult to identity from this data only whether the obtained level of similarity is due to natural variety in human behaviours, or to the difference in conditions explored in each block, we propose to measure similarity between paths belonging to the same block as follows. For each block and each configuration, we randomly divided the trajectories into two subsets and computed the Dice similarity score between them. We repeated this process 30 times (which changes the way trajectories are divided into 2 subsets). Performing this process and computing similarity over the 3 blocks resulted into 90 measures of "intra-block similarity". The obtained average value is $81.2 \pm 3.3 \%$, that can be compared with the "inter-block similarity" scores presented in Table 1.

Our results show that there is no statistical difference between intra-block and Nohaptic1 vs. Haptic blocks similarity measure $(p>0.05)$. There is however a significant difference between intra-block and Haptic vs. Nohaptic2 blocks $(p<0.01)$, as well as intra-block and Nohaptic1 vs. Nohaptic2 $(p<0.05)$, where intra-block similarity measures are always lower. Given that similarity measures between pairs of blocks were either as similar or more similar than intra-block similarities, we can conclude that participants chose their path through the crowd similarly, irrespective of the block condition, which supports $H 1$. To better illustrate the similarity in navigation paths, Figure 10 displays all the participants' trajectories and the triangles used to compute the Dice for the specific $T_{6}$ configuration.

\subsection{Body Motion}

\subsubsection{Shoulder Rotation}

The average amplitude of shoulder rotations $\alpha_{S A}$, illustrated in Figure 11.a, was significantly different in each block $\left(F(2,44)=13.0, p<0.001, \eta_{p}^{2}=0.37\right)$. In particular, it was significantly higher in the block with haptic rendering $\left(40.1 \pm 8.2^{\circ}\right)$, than in the first block without haptic rendering $\left(34.3 \pm 6.0^{\circ}\right)$. We remind that a higher $\alpha_{S A}$ angle means that participants made a larger rotation to squeeze
TABLE 1

Similarity measure (Dice) of participant trajectories between all blocks (NoHaptic1, Haptic, NoHaptic2) for all the trials.

\begin{tabular}{|l|c|c|c|}
\hline \multirow{2}{*}{ Trials } & \multicolumn{3}{|c|}{ Blocks } \\
& NoHaptic1 vs. Haptic & Haptic vs. NoHaptic2 & NoHaptic1 vs. NoHaptic2 \\
\hline$T_{1}$ & $84.0 \%$ & $88.6 \%$ & $85.0 \%$ \\
\hline$T_{2}$ & $88.4 \%$ & $93.8 \%$ & $88.3 \%$ \\
\hline$T_{3}$ & $78.1 \%$ & $93.2 \%$ & $79.4 \%$ \\
\hline$T_{4}$ & $91.9 \%$ & $88.7 \%$ & $90.7 \%$ \\
\hline$T_{5}$ & $88.4 \%$ & $90.2 \%$ & $85.3 \%$ \\
\hline$T_{6}$ & $82.8 \%$ & $85.8 \%$ & $91.0 \%$ \\
\hline$T_{7}$ & $78.8 \%$ & $81.6 \%$ & $82.0 \%$ \\
\hline$T_{8}$ & $85.0 \%$ & $85.9 \%$ & $85.3 \%$ \\
\hline$T_{\text {all }}$ & $\mathbf{8 4 . 7} \pm \mathbf{4 . 8 \%}$ & $\mathbf{8 8 . 6} \pm \mathbf{4 . 1 \%}$ & $\mathbf{8 5 . 9} \pm \mathbf{4 . 0 \%}$ \\
\hline
\end{tabular}

between virtual characters, therefore validating the hypotheses $H 2_{1}$. Furthermore, it was also significantly higher in block NoHaptic2 $\left(38.7 \pm 3.7^{\circ}\right)$ than in block NoHaptic1, suggesting that participants continued to turn more their shoulders even after haptic rendering was disabled, therefore supporting $H 3$.

\subsubsection{Walking Speed}

We found an effect of haptic rendering $(F(1.56,34.2)=$ $\left.7.14, p=0.005, \eta_{p}^{2}=0.245\right)$ on participant's average walking speed (Figure 11.b), where participants' walking speed was on average significantly lower in theHaptic block $(0.40 \pm 0.07 \mathrm{~m} / \mathrm{s})$ than in the NoHaptic1 $(0.43 \pm 0.07 \mathrm{~m} / \mathrm{s})$ and NoHaptic2 $(0.42 \pm 0.07 \mathrm{~m} / \mathrm{s})$ blocks. This result therefore supports hypothesis $H 2_{1}$.

\subsection{Collisions}

Figures 11.c and 11.d illustrate the results regarding collision characteristics, i.e., number of collisions as well as volume of interpenetration.

The average number of collisions per trial was influenced by haptic rendering with a large effect $(F(2,44)=$ $7.13, p=0.002, \eta_{p}^{2}=0.25$ ). Post-hoc analysis showed that the number of collisions was higher during the NoHaptic1 block $(71 \pm 29.2)$ than during the Haptic $(62.8 \pm 34.6$, $p=0.018)$ and NoHaptic2 blocks $(60.7 \pm 34.6, p=0.002)$, which shows that participants made on average more collisions before they experienced haptic rendering. 


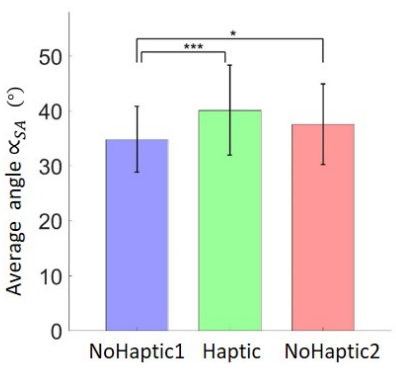

a)

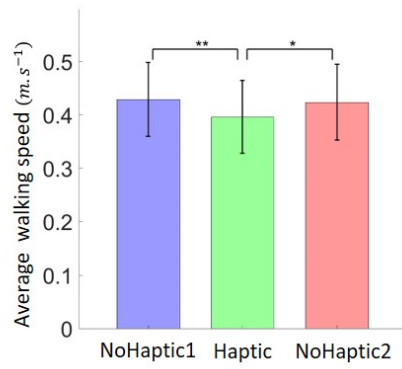

b)

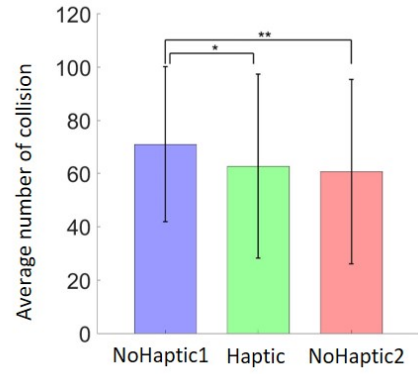

c)

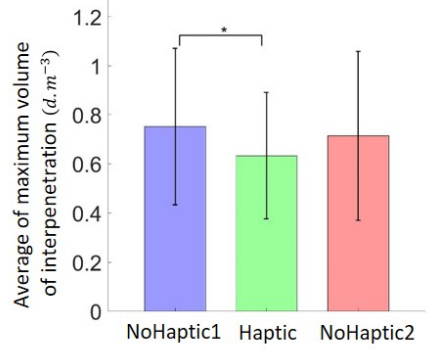

d)

Fig. 11. Main significant differences between the three blocks of the experiment (NoHaptic1, Haptic and NoHaptic2): a) amplitude of shoulder rotations $\left(\alpha_{S A}\right)$, b) walking speed, c) number of collisions per trial, d) volume of interpenetration. Error bars depict standard deviation of the mean.

The average volume of interpenetration was also influenced by the block $\left(F(2,44)=4.35, p=0.019, \eta_{p}^{2}=0.16\right)$, where post-hoc analysis showed that this volume was smaller $(p=0.016)$ in the Haptic block $\left(0.6 \pm 0.3 \mathrm{dm}^{-3}\right)$ than during the NoHaptic1 $\left(0.8 \pm 0.3 \mathrm{dm}^{-3}\right)$.

These results validate our hypothesis $H 2_{2}$, that states that haptic rendering reduces the severity of collisions between participants and virtual characters. Furthermore, as the number of collisions is higher during block NoHaptic1 than during block NoHaptic2, this also supports $H 3$ on potential after-effects of haptic rendering.

\subsection{Presence and Embodiment}

The average participant ratings and all the questions for embodiment are shown in Tables 2,3 and 4. We did not find any significant effect of the blocks for Agency $(p=0.438)$, Change ( $p=0.085)$ and Ownership $(p=0.753)$. Furthermore, Table 5 shows the questions and the average participant ratings for presence, for which we also did not find a significant effect of the blocks $(p=0.222)$. These results therefore do not support hypothesis $H 4$, suggesting that haptic rendering does not improve the sense of presence or the sense of embodiment of participants in VR.

TABLE 2

Agency questionnaire: average participant ratings for the three blocks.

\begin{tabular}{|l|l|l|l|}
\hline Questions & NoHaptic1 & Haptic & NoHaptic2 \\
\hline $\begin{array}{l}\text { The movements of the virtual body felt like } \\
\text { they were my movements. }\end{array}$ & & & \\
$\begin{array}{l}\text { I felt like I was controlling the movements } \\
\text { of the virtual body }\end{array}$ & & & \\
$\begin{array}{l}\text { I felt like I was causing the movements of } \\
\text { the virtual body. } \\
\text { The movements of the virtual body were in } \\
\text { sync with my own movements. }\end{array}$ & $6.1 \pm 0.9$ & $6.0 \pm 0.8$ & $5.9 \pm 0.7$ \\
\hline
\end{tabular}

\section{TABLE 3}

Change questionnaire: average participant ratings for the three blocks.

\begin{tabular}{|l|c|c|c|}
\hline Questions & NoHaptic1 & $\begin{array}{c}\text { Blocks } \\
\text { Haptic }\end{array}$ & NoHaptic2 \\
\hline $\begin{array}{l}\text { I felt like the form or appearance of my own } \\
\text { body had changed. }\end{array}$ & & & \\
$\begin{array}{l}\text { It felt like the weight of my own body had } \\
\text { changed. }\end{array}$ & $3.6 \pm 1.3$ & $3.8 \pm 1.5$ & $3.3 \pm 1.5$ \\
$\begin{array}{l}\text { I felt like the size (height) of my own body } \\
\text { had changed. }\end{array}$ & & & \\
$\begin{array}{l}\text { I felt like the width of my own body had } \\
\text { changed. }\end{array}$ & & & \\
\hline
\end{tabular}

TABLE 4

Ownership questionnaire: average participant ratings for the three blocks.

\begin{tabular}{|l|c|c|c|}
\hline Questions & NoHaptic1 & Haptic & NoHaptic2 \\
\hline $\begin{array}{l}\text { It felt like the virtual body was my body. } \\
\text { It felt like the virtual body parts were } \\
\text { my body parts. }\end{array}$ & & & \\
$\begin{array}{l}\text { The virtual body felt like a human body. } \\
\text { It felt like the virtual body belonged to me. }\end{array}$ & $4.9 \pm 1.4$ & $5.1 \pm 1.2$ & $5.0 \pm 1.2$ \\
\hline
\end{tabular}

TABLE 5

Slater-Usoh-Steed (SUS) questionnaire [48] and average participant ratings for the three blocks.

\begin{tabular}{|l|l|l|l|}
\hline Questions & NoHaptic1 & \multicolumn{1}{|c|}{ Haptic } & NoHaptic2 \\
\hline $\begin{array}{l}\text { I had a sense of being there in the train station. } \\
\text { There were times during the experience when... } \\
\text { the train station was the reality for me... }\end{array}$ & & & \\
$\begin{array}{l}\text { The train station seems to me to be more like... } \\
\text { I had a stronger sense of... }\end{array}$ & $5.2 \pm 0.9$ & $5.2 \pm 1.2$ & $5.0 \pm 1.1$ \\
$\begin{array}{l}\text { I think of the train station as a place in a way } \\
\text { similar to other places that I've been today. } \\
\text { During the experience I often thought that I } \\
\text { was really standing in the train station. }\end{array}$ & & & \\
\end{tabular}

\section{Discussion}

The main objective of this study was to evaluate the effect of haptic rendering of collisions on participants' behaviour while navigating in a dense virtual crowd. To this end, we designed an experiment where participants had to reach a goal by physically walking in a virtual train station populated with a dense crowd. Participants were equipped with vibrotactile sensors located on their arms and performed this task following 3 blocks: NoHaptic1, Haptic and NoHaptic2, for which haptic rendering of collisions with virtual characters was not experienced, experienced, and not experienced again, respectively.

\subsection{Trajectories}

In Section 5.1, the analysis of the Dice similarity measure showed that haptic rendering did not change the way participants selected their path through the crowd, as stated in hypothesis $H 1$. We even found that paths across blocks were "more similar" than within the same block. One possible explanation is given by the way we compose the sets we compare the similarity of, where we assume that paths are independent from participants. Indeed, the intra-block similarity measure required us to split a set of trajectories belonging to the same block and crowd configuration, 
which resulted into comparing paths performed by different participants. In contrast, the inter-block analysis considered sets that were split according to haptic rendering conditions, thus comparing paths performed by the same group of 23 participants.

In spite of this limitation in our analysis, we consider that paths are similar across blocks. One can describe human motion as a trajectory resulting from a perception-action loop [21], [50]. Depending on the tasks, the loop is a multimodal one, meaning that different senses are used to control motion. However in the context of walking, several studies [37], [50] have shown that vision is the most used perceptual input to navigate to the goal. Such statements hold in our case, where a major difference with previous work is the higher density of obstacles. Nevertheless, assuming that tactile feedback may affect path selection, it would have been probable that some participants reversed their course after a collision has been rendered, which was not observed.

\subsection{Avoidance Behaviour}

In this experiment, we demonstrated that haptic rendering had an effect on shoulder rotations, which supports hypothesis $H 2_{1}$. In particular, participants rotated more their shoulders when traversing the gaps between virtual characters during the Haptic block than during the NoHaptic1 block. This result is consistent with the observations of Mestre et al. [32] with participants passing through a virtual halfopen door with or without haptic rendering. More generally, let us remind that the human trunk is most often larger along the transverse axis than along the antero-posterior axis. Thus, the more the participants turn their shoulders the smaller the volume swept by their body motion. Our results therefore suggest that participants might have tried to minimize the risk of collision with virtual characters more in the condition where they experienced haptic rendering than in the first block of the experiment. The slower speed observed in the Haptic block also reveals that participants moved more cautiously.

Being more cautious effectively resulted into less collisions as expected in hypothesis $H 2_{2}$. Results presented in Section 5.3 show that the average number of collisions as well as the average volume of interpenetration were significantly lower in the Haptic block than in the NoHaptic1 block. Furthermore, this observation is consistent with previous studies [30] where haptic feedback lowered the number of collisions with a static object.

\subsection{Haptic Rendering After-effects}

While there were less collisions and more shoulder rotations observed in the Haptic block in comparison with the NoHaptic1 block, there was no difference between the Haptic and the NoHaptic2 blocks. This supports hypothesis $H 3$ on potential after-effects of haptic rendering. However, such an after-effect did not equally influence all measurements, such as walking speed that increased again in the NoHaptic2 block. One possible explanation might be a perceptual calibration of the participants. During the experiment, participants became more familiar with the environment, the task to be performed, but also the virtual representation of their body and the virtual environment, enabling them to move faster and better avoid collisions with the virtual characters in the last block (NoHaptic2). Another point to highlight is that participants, at the beginning of the Haptic block, did not know that contacts would now trigger a vibrotactile haptic sensation. For this reason, we might expect to see a short learning phase at the beginning of the block, where participants learn to deal with the newly-rendered haptic collisions. Considering this point, we can expect the effect of providing haptic sensations of collisions even stronger than registered. However, to provide a more definitive conclusion on the role of the haptic after-effect would require to add a control group with no haptic rendering throughout the 3 blocks of the experiment, which could be explored in future work.

These results can also open perspectives regarding the design of new experiments including haptic priming tasks. In a recent study, Krum et al. [27] showed that haptic priming of collision had no effect on participants' proxemics and more precisely on distances with a virtual character. It is important to note that the task was different: it included an interaction with one virtual character and there were no risks of collision since the virtual character never came very close to the participant. It would be interesting then to reevaluate such influence when the intimate space is violated by a virtual character.

\subsection{Embodiment and Presence}

In contrast with our hypothesis $H 4$, we did not find any significant change in terms of user's perceived senses of embodiment and presence when experiencing haptic feedback. This result is quite surprising, as we did find significant effects in other measurements, suggesting that participants took different actions when provided with haptic sensations of contact. An explanation for this result could lie in the fact that users already registered high embodiment and presence levels without experiencing haptic feedback in the first condition (NoHaptic1), leaving little room for improvement in the Haptic condition. Another possibility is that vibrotactile feedback is not suited to render collisions in crowds, although there are several examples of this type of feedback being used to render similar events [5], [18]. Finally, a last explanation could be the location and number of our haptic devices. Employing a higher number of bracelets spread throughout the body might better render the target contact sensations. All these considerations will drive our future work.

\subsection{Limitations}

Our study had a few limitations. As explained above, we employed a limited number of haptic rendering devices located on participants arms only. It is quite possible that employing more devices, including some for the legs and hips, would have resulted in stronger effects. However, our setup still revealed significant effects, and the question of nature, number, and location of haptic devices would probably require a fully dedicated study. Another related issue is the quality of the provided haptic sensations. Our devices show high wearability and portability, but can only provide vibrotactile haptic sensations. Other haptic delivery options include the use of arm or full-body exoskeletons, which 
can provide well-rounded force sensations. However, these devices are significantly more cumbersome and expensive that those employed in this work, severely limiting their applicability and availability.

A second limitation concerns the behaviour of the virtual characters present in the crowd. Indeed, they do not react to collisions, as noticed by some participants in their feedback. It would therefore be required to have an animation technique capable of reacting to collisions such as, for instance, the virtual character taking a step in the opposite direction of the collision. We could also trigger verbal reactions to express that virtual characters are embarrassed by collisions. Adding such virtual behaviours combined with haptic feedback could improve participants' immersion and feeling of presence.

Finally, one last point concerns the many devices (armbands, MSI VR one, HMD, X-Sens, etc.) required to be worn by participants for a significant amount of time. Carrying such equipment can have an effect on participants' motion as well as comfort. In our case, the experience was still relatively short and lasted only for 15 to 20 minutes. However, longer immersion durations might require to use wireless HMD solutions instead, even if this today means decreasing the field of vision.

\section{Conclusion}

In this paper, we designed an experiment to evaluate the effects, as well as the after-effects, of haptic rendering on a motion task in a highly crowded environment. Participants performed a goal-directed navigation task through a dense virtual crowd. Wearable haptic devices provided them with vibrotactile feedback whenever a collision with their arms occurred. Results showed that providing haptic feedback impacted the way participants moved through the virtual crowd. They were more cautious about the collisions they provoked with virtual characters, but they did not change their global trajectories. We also demonstrated the presence of an after-effect of haptic feedback, since changes in their movements remained after haptic feedback was disabled. Finally, quite surprisingly, we did not notice any impact of haptic rendering on the perceived Presence and Embodiment. These results show that visual information is probably the main sense used for navigation in dense crowds. However, a combination of visual and haptic feedback improves the overall realism of the experience, as participants show a more realistic behaviour: they are more cautious about not touching virtual characters. For this reason, we therefore suggest using haptic rendering to study human behaviour and locomotion interactions that may lead to contacts.

For future work, we are interested in populating our virtual environments with more interactive and reactive virtual characters. This is a crucial aspect since it seems to be a requirement to further improve the feeling of presence of participants. Also, the use of reactive characters may increase the effect of haptic rendering, since we could expect stronger participant reactions when virtual characters would also react after a collision. A more detailed analysis that evaluates motion before and after a collision is rendered and a virtual character reacts would then also be relevant to study. We are also interested in carrying out experiments enrolling more subjects and analysing a wider range of metrics in different scenarios (e.g., considering a dynamic crowd, measuring the effect on shoulder hunching, carrying out a control experiment where no haptics is applied).Finally, we plan to use more compelling wearable haptic devices to provide a more realistic sensation of collision while keeping the overall system compact and easy to wear, e.g., skin stretch [13] or tapping devices for the shoulder and upper arm.

\section{ACKNOWLEDGMENTS}

This project has received funding from the European Union's Horizon 2020 research and innovation programme under grant agreement No 779942 Crowdbot and No 856879 PRESENT, as well as from the ANR OPMoPS project (ANR16-SEBM-0004).

\section{REFERENCES}

[1] J. Achenbach, T. Waltemate, M. E. Latoschik, and M. Botsch. Fast generation of realistic virtual humans. In Proceedings of the 23rd ACM Symposium on Virtual Reality Software and Technology, pp. 1$10,2017$.

[2] M. Aggravi, F. Pausé, P. R. Giordano, and C. Pacchierotti. Design and evaluation of a wearable haptic device for skin stretch, pressure, and vibrotactile stimuli. IEEE Robotics and Automation Letters, 3(3):2166-2173, 2018.

[3] F. Berton, L. Hoyet, A.-H. Olivier, J. Bruneau, O. Le Meur, and J. Pettré. Eye-gaze activity in crowds: Impact of virtual reality and density. In 2020 IEEE Conference on Virtual Reality and 3D User Interfaces (VR). IEEE, 2020.

[4] F. Berton, A.-H. Olivier, J. Bruneau, L. Hoyet, and J. Pettré Studying gaze behaviour during collision avoidance with a virtual walker: Influence of the virtual reality setup. In 2019 IEEE Conference on Virtual Reality and 3D User Interfaces (VR), pp. 717725. IEEE, 2019.

[5] J. Bimbo, C. Pacchierotti, M. Aggravi, N. Tsagarakis, and D. Prattichizzo. Teleoperation in cluttered environments using wearable haptic feedback. In Proc. IEEE/RSJ International Conference on Intelligent Robots and Systems (IROS), pp. 3401-3408, 2017.

[6] S. Bonneaud, K. Rio, P. Chevaillier, and W. H. Warren. Accounting for patterns of collective behavior in crowd locomotor dynamics for realistic simulations. In Transactions on Edutainment VII, pp. 1-11. Springer, 2012.

[7] A. Bönsch, S. Radke, H. Overath, L. M. Asché, J. Wendt, T. Vierjahn, U. Habel, and T. W. Kuhlen. Social vr: How personal space is affected by virtual agents' emotions. In 2018 IEEE Conference on Virtual Reality and 3D User Interfaces (VR), pp. 199-206, 2018.

[8] S. M. Bourgaize, B. J. McFadyen, and M. E. Cinelli. Collision avoidance behaviours when circumventing people of different sizes in various positions and locations. Journal of Motor Behavior, pp. 1-10, 2020.

[9] J. Bruneau, A.-H. Olivier, and J. Pettre. Going through, going around: A study on individual avoidance of groups. IEEE transactions on visualization and computer graphics, 21(4):520-528, 2015.

[10] U. Chattaraj, A. Seyfried, and P. Chakroborty. Comparison of pedestrian fundamental diagram across cultures. Advances in complex systems, 12(03):393-405, 2009.

[11] L. P. Chew. Constrained delaunay triangulations. Algorithmica, 4(1-4):97-108, 1989.

[12] F. Chinello, M. Malvezzi, D. Prattichizzo, and C. Pacchierotti. A modular wearable finger interface for cutaneous and kinesthetic interaction: control and evaluation. IEEE Transactions on Industrial Electronics, 67(1):706-716, 2019.

[13] F. Chinello, C. Pacchierotti, J. Bimbo, N. G. Tsagarakis, and D. Prattichizzo. Design and evaluation of a wearable skin stretch device for haptic guidance. IEEE Robotics and Automation Letters, 3(1):524531, 2017. 
[14] F. Chinello, C. Pacchierotti, M. Malvezzi, and D. Prattichizzo. A three revolute-revolute-spherical wearable fingertip cutaneous device for stiffness rendering. IEEE Transactions on Haptics, 11(1):3950, 2017.

[15] S. Cho, J. Park, and O. Kwon. Gender differences in three dimensional gait analysis data from 98 healthy korean adults. Clinical biomechanics, 19(2):145-152, 2004.

[16] G. Cirio, A.-H. Olivier, M. Marchal, and J. Pettre. Kinematic evaluation of virtual walking trajectories. IEEE transactions on visualization and computer graphics, 19(4):671-680, 2013.

[17] J. L. Croft and D. Panchuk. Watch where you're going? interferer velocity and visual behavior predicts avoidance strategy during pedestrian encounters. Journal of motor behavior, 50(4):353-363, 2018.

[18] L. Devigne, M. Aggravi, M. Bivaud, N. Balix, S. Teodorescu, T. Carlson, T. Spreters, C. Pacchierotti, and M. Babel. Power wheelchair navigation assistance using wearable vibrotactile haptics. IEEE Transactions on Haptics, 2020.

[19] P. Dickinson, K. Gerling, K. Hicks, J. Murray, J. Shearer, and J. Greenwood. Virtual reality crowd simulation: effects of agent density on user experience and behaviour. Virtual Reality, 23(1):1932, 2019.

[20] C. Faure. Vers des environnements virtuels plus écologiques: étude des modifications du comportement moteur en réalité virtuelle lors de l'ajout d'informations haptiques par un mécanisme parallèle entraîné par câbles. 2019.

[21] J. J. Gibson. Visually controlled locomotion and visual orientation in animals. British journal of psychology, 49(3):182-194, 1958.

[22] R. S. Hessels, A. J. van Doorn, J. S. Benjamins, G. A. Holleman, and I. T. Hooge. Task-related gaze control in human crowd navigation. Attention, Perception, \& Psychophysics, pp. 1-20, 2020.

[23] P.-H. Huang and S.-K. Wong. Emotional virtual crowd on task completion in virtual markets. Computer Animation and Virtual Worlds, 29(3-4):e1818, 2018.

[24] M. Huber, Y.-H. Su, M. Krüger, K. Faschian, S. Glasauer, and J. Hermsdörfer. Adjustments of speed and path when avoiding collisions with another pedestrian. PloS one, 9(2), 2014.

[25] A. G. Knorr, L. Willacker, J. Hermsdörfer, S. Glasauer, and M. Krüger. Influence of person-and situation-specific characteristics on collision avoidance behavior in human locomotion. Journal of experimental psychology: human perception and performance, 42(9):1332, 2016.

[26] C. Krogmeier, C. Mousas, and D. Whittinghill. Human, virtual human, bump! a preliminary study on haptic feedback. In 2019 IEEE Conference on Virtual Reality and 3D User Interfaces (VR), pp. 1032-1033, 2019.

[27] D. M. Krum, S.-H. Kang, and T. Phan. Influences on the elicitation of interpersonal space with virtual humans. In 2018 IEEE Conference on Virtual Reality and 3D User Interfaces (VR), pp. 223-9, 2018.

[28] R. W. Lindeman, R. Page, Y. Yanagida, and J. L. Sibert. Towards full-body haptic feedback: the design and deployment of a spatialized vibrotactile feedback system. In Proceedings of the ACM symposium on Virtual reality software and technology, pp. 146-149, 2004.

[29] R. W. Lindeman, Y. Yanagida, H. Noma, and K. Hosaka. Wearable vibrotactile systems for virtual contact and information display. Virtual Reality, 9(2-3):203-213, 2006.

[30] C. Louison, F. Ferlay, and D. R. Mestre. Spatialized vibrotactile feedback improves goal-directed movements in cluttered virtual environments. International Journal of Human-Computer Interaction, 34(11):1015-1031, 2018.

[31] S. D. Lynch, R. Kulpa, L. A. Meerhoff, J. Pettre, A. Cretual, and A.-H. Olivier. Collision avoidance behavior between walkers: global and local motion cues. IEEE transactions on visualization and computer graphics, 24(7):2078-2088, 2017.

[32] D. R. Mestre, C. Louison, and F. Ferlay. The contribution of a virtual self and vibrotactile feedback to walking through virtual apertures. In International Conference on Human-Computer Interaction, pp. 222-232. Springer, 2016.

[33] A.-H. Olivier, J. Bruneau, R. Kulpa, and J. Pettré. Walking with virtual people: Evaluation of locomotion interfaces in dynamic environments. IEEE transactions on visualization and computer graphics, 24(7):2251-2263, 2017.

[34] A.-H. Olivier, A. Marin, A. Crétual, A. Berthoz, and J. Pettré. Collision avoidance between two walkers: Role-dependent strategies. Gait E posture, 38(4):751-756, 2013.
[35] A.-H. Olivier, A. Marin, A. Crétual, and J. Pettré. Minimal predicted distance: A common metric for collision avoidance during pairwise interactions between walkers. Gait E posture, 36(3):399404, 2012.

[36] C. Pacchierotti, S. Sinclair, M. Solazzi, A. Frisoli, V. Hayward, and D. Prattichizzo. Wearable haptic systems for the fingertip and the hand: taxonomy, review, and perspectives. IEEE Transactions on Haptics, 10(4):580-600, 2017.

[37] A. E. Patla. Understanding the roles of vision in the control of human locomotion. Gait \& Posture, 5(1):54-69, 1997.

[38] K. Rio and W. H. Warren. The visual coupling between neighbors in real and virtual crowds. Transportation Research Procedia, 2:132140, 2014.

[39] K. W. Rio, G. C. Dachner, and W. H. Warren. Local interactions underlying collective motion in human crowds. Proceedings of the Royal Society B: Biological Sciences, 285(1878):20180611, 2018.

[40] D. Roth and M. E. Latoschik. Construction of a validated virtual embodiment questionnaire. arXiv preprint arXiv:1911.10176, 2019.

[41] S. V. Salazar, C. Pacchierotti, X. de Tinguy, A. Maciel, and M. Marchal. Altering the stiffness, friction, and shape perception of tangible objects in virtual reality using wearable haptics. IEEE transactions on haptics, 13(1):167-174, 2020.

[42] S. Scheggi, M. Aggravi, and D. Prattichizzo. Cooperative navigation for mixed human-robot teams using haptic feedback. IEEE Transactions on Human-Machine Systems, 47(4):462-473, 2016.

[43] S. B. Schorr and A. M. Okamura. Three-dimensional skin deformation as force substitution: Wearable device design and performance during haptic exploration of virtual environments. IEEE transactions on haptics, 10(3):418-430, 2017.

[44] A. Seyfried, B. Steffen, W. Klingsch, and M. Boltes. The fundamental diagram of pedestrian movement revisited. Journal of Statistical Mechanics: Theory and Experiment, 2005(10):P10002, 2005.

[45] W. S. Silva, G. Aravind, S. Sangani, and A. Lamontagne. Healthy young adults implement distinctive avoidance strategies while walking and circumventing virtual human vs. non-human obstacles in a virtual environment. Gait $\mathcal{E}$ posture, 61:294-300, 2018.

[46] M. Slater. A note on presence terminology. Presence connect, 3(3):15, 2003.

[47] T. J. Sørensen. A method of establishing groups of equal amplitude in plant sociology based on similarity of species content and its application to analyses of the vegetation on Danish commons. I kommission hos E. Munksgaard, 1948.

[48] M. Usoh, E. Catena, S. Arman, and M. Slater. Using presence questionnaires in reality. Presence: Teleoperators \& Virtual Environments, 9(5):497-503, 2000.

[49] M. Volonte, Y. C. Hsu, K.-y. Liu, J. Mazer, S.-K. Wong, and S. Babu. Effects of interacting with a crowd of emotional virtual humans on users' affective and non-verbal behaviors. In 2020 IEEE Conference on Virtual Reality and 3D User Interfaces (VR). IEEE, 2020.

[50] W. Warren. Visually controlled locomotion: 40 years later. Ecological Psychology, 10(3-4):177-219, 1998.

[51] K. Wilmut and A. L. Barnett. Locomotor adjustments when navigating through apertures. Human Movement Science, 29(2):289298,2010 . 